\title{
Sharpness and non-compactness of embeddings of Bessel-potential-type spaces
}

\author{
Amiran Gogatishvili ${ }^{* 1}$, Júlio Severino Neves ${ }^{* * 2}$, and Bohumír Opic ${ }^{* * * 1}$ \\ ${ }^{1}$ Mathematical Institute, Academy of Sciences of the Czech Republic, Žitná 25, 11567 Prague 1, Czech Republic \\ ${ }^{2}$ CMUC, Department of Mathematics, University of Coimbra, Apartado 3008, 3001-454 Coimbra, Portugal
}

Received 10 December 2005, revised 21 July 2006, accepted 29 July 2006

Published online 6 June 2007

Key words Slowly varying functions, Lorentz-Karamata spaces, rearrangement-invariant Banach function spaces, (fractional) Sobolev-type spaces, Hölder-type spaces, Bessel potentials, embedding theorems

MSC (2000) 46E35, 46E30, 26D 15

Dedicated to Professor Frank-Olme Speck on the occasion of his 60th birthday

We establish embeddings for Bessel potential spaces modeled upon Lorentz-Karamata spaces with order of smoothness less than one. The target spaces are of Hölder-continuous type. In the super-limiting case we also prove that the embedding is sharp and fails to be compact.

(c) 2007 WILEY-VCH Verlag GmbH \& Co. KGaA, Weinheim

\section{Introduction}

In a series of recent papers [7]-[10] a systematic research of embeddings of Bessel potential spaces with order of smoothness $\sigma \geq 1$ and modeled upon generalized Lorentz-Zygmund (GLZ) spaces was carried out. The authors of those papers established embeddings of such spaces either into GLZ-spaces or into Hölder-type spaces $C^{0, \lambda(\cdot)}(\bar{\Omega})$ and showed that their results are sharp (within the given scale of target spaces) and fail to be compact. They also clarified the role of the logarithmic terms involved in the quasi-norms of the spaces mentioned. This role proved to be important especially in limiting cases. In particular, they obtained refinements of the Sobolev embedding theorems, Trudinger's limiting embedding as well as embeddings of Sobolev spaces into $\lambda(\cdot)$-Hölder continuous functions including the result of Brézis and Wainger about almost Lipschitz continuity of elements of the (fractional) Sobolev space $H_{p}^{1+n / p}\left(\mathbf{R}^{n}\right)$ (cf. [5]).

Although GLZ-spaces form an important scale of spaces containing, for example, Zygmund classes $L^{p}(\log L)^{\alpha}$, Orlicz spaces of multiple exponential type, Lorentz spaces $L_{p, q}$, Lebesgue spaces $L_{p}$, etc., they are a particular case of more general spaces, namely the Lorentz-Karamata (LK) spaces.

The embeddings mentioned above were extended in [20] and [21] to the case when Bessel-potential spaces are modeled upon LK-spaces. Since Neves considered more general targets (besides LK-spaces and Hölder-type spaces also generalized Hölder spaces), in several cases he obtained improvements of embeddings from [7]-[10]. The sharpness and non-compactness of these embeddings were proved in [15] and [16].

In [11] and [12], the authors analyzed the situation when the order of smoothness is less than one. In such a case one cannot use the method in which a lifting argument (based on [9, Lemma 4.1] and [16, Lemma 4.5], which extend the Calderón result [6, Theorem 7]) is applied to reduce the superlimiting case to the sublimiting one, and a new approach was used.

Although many results were obtained, the research is not yet complete. Here we extend some results of [11] and [12]. Nevertheless, there are still open questions which are under investigation.

* e-mail: gogatish@math.cas.cz, Phone: +420222090786, Fax: +420222211638

** Corresponding author: e-mail: jsn@mat.uc.pt, Phone: +35 1239791150, Fax: +35 1239793069

*** e-mail: opic@math.cas.cz, Phone: +420222090745, Fax: +420222211638 
The paper is organized as follows. Section 2 contains notation, definitions and basic properties, while the main results are stated in Section 3. After some preliminaries in the next two sections, the final Section 6 gives the proofs of the promised theorems.

\section{Notation, definitions and basic properties}

As usual, $\mathbf{R}^{n}$ denotes the Euclidean $n$-dimensional space. Throughout the paper, $\mu_{n}$ is the $n$-dimensional Lebesgue measure in $\mathbf{R}^{n}$ and $\Omega$ is a $\mu_{n}$-measurable subset of $\mathbf{R}^{n}$. We denote by $\chi_{\Omega}$ the characteristic function of $\Omega$ and write $|\Omega|_{n}=\mu_{n}(\Omega)$. The family of all extended scalar-valued (real or complex) $\mu_{n}$-measurable functions on $\Omega$ will be denoted by $\mathcal{M}(\Omega)$. The non-increasing rearrangement of $f \in \mathcal{M}(\Omega)$ is the function $f^{*}$ defined by $f^{*}(t)=\inf \left\{\lambda \geq 0:|\{x \in \Omega:|f(x)|>\lambda\}|_{n} \leq t\right\}$ for all $t \geq 0$.

Given a rearrangement-invariant Banach function space (r.i. BFS) $X$, the associate space is denoted by $X^{\prime}$. For general facts about (rearrangement-invariant) Banach function spaces we refer to [3, Chaps. $1 \& 2$ ]

Let $X$ and $Y$ be two (quasi-)Banach spaces. We say that $X$ coincides with $Y$ (and write $X=Y$ ) if $X$ and $Y$ are equal in the algebraic and topological sense (their (quasi-)norms are equivalent). The symbol $X \hookrightarrow Y$ means that $X \subset Y$ and the natural embedding of $X$ into $Y$ is continuous.

By $c, c_{1}, c_{2}$, etc. we denote positive constants independent of appropriate quantities. For two nonnegative expressions (i.e. functions or functionals) $\mathcal{A}, \mathcal{B}$, the symbol $\mathcal{A} \precsim \mathcal{B}$ (or $\mathcal{A} \succsim \mathcal{B}$ ) means that $\mathcal{A} \leq c \mathcal{B}$ (or $c \mathcal{A} \geq \mathcal{B}$ ). If $\mathcal{A} \precsim \mathcal{B}$ and $\mathcal{A} \succsim \mathcal{B}$, we write $\mathcal{A} \approx \mathcal{B}$ and say that $\mathcal{A}$ and $\mathcal{B}$ are equivalent. We adopt the convention that $a /+\infty=0$ and $a / 0=+\infty$ for all $a>0$. If $p \in[1,+\infty]$, the conjugate number $p^{\prime}$ is given by $1 / p+1 / p^{\prime}=1$.

For $\rho \in(0,+\infty)$ and $x \in \mathbf{R}^{n}, B_{n}(x, \rho)$ stands for the open ball in $\mathbf{R}^{n}$ of radius $\rho$ and centre $x$. By $\omega_{n}$ we denote the volume of the unit ball in $\mathbf{R}^{n}$.

Following [17], we say that a positive and Lebesgue-measurable function $b$ is slowly varying on $(0,+\infty)$, and write $b \in S V(0,+\infty)$, if, for each $\epsilon>0, t^{\epsilon} b(t)$ is equivalent to a non-decreasing function on $(0,+\infty)$ and $t^{-\epsilon} b(t)$ is equivalent to a non-increasing function on $(0,+\infty)$. The family of all slowly varying functions includes not only powers of iterated logarithms and the broken logarithmic functions of [14], but also such functions as $t \rightarrow \exp \left(|\log t|^{a}\right), a \in(0,1)$. (The last mentioned function has the interesting property that it tends to infinity more quickly than any positive power of the logarithmic function.)

It can be shown (cf. [17]) that any $b \in S V(0,+\infty)$ is equivalent to a $\widetilde{b} \in S V(0,+\infty)$ which is continuous on $(0,+\infty)$. Consequently, without loss of generality, we shall assume that all slowly varying functions in question are continuous on $(0,+\infty)$.

More properties and examples of slowly varying functions can be found in [4], [13], [17], [18], [20] and [22, Chap. V, p. 186].

Let $p, q \in(0,+\infty]$ and $b \in S V(0,+\infty)$. The Lorentz-Karamata $(\mathrm{LK})$ space $L_{p, q ; b}(\Omega)$ is defined to be the set of all functions $f \in \mathcal{M}(\Omega)$ such that $\|f\|_{p, q ; b ; \Omega}:=\left\|t^{1 / p-1 / q} b(t) f^{*}(t)\right\|_{q ;(0,+\infty)}$ is finite. Here $\|\cdot\|_{q ;(0,+\infty)}$ stands for the usual $L_{q}$-(quasi-)norm on the interval $(0,+\infty)$.

When $0<p<+\infty$, the Lorentz-Karamata space $L_{p, q ; b}(\Omega)$ contains the characteristic function of every measurable subset of $\Omega$ with finite measure and hence, by linearity, every $\mu_{n}$-simple function. When $p=+\infty$, the Lorentz-Karamata space $L_{p, q ; b}(\Omega)$ is different from the trivial space if, and only if, $\left\|t^{1 / p-1 / q} b(t)\right\|_{q ;(0,1)}<$ $+\infty$.

Particular choices of $b$ give well-known spaces. If $m \in \mathbf{N}, \boldsymbol{\alpha}=\left(\alpha_{1}, \ldots, \alpha_{m}\right) \in \mathbf{R}^{m}$ and $b=\boldsymbol{\ell}^{\boldsymbol{\alpha}}$, where

$$
\ell^{\boldsymbol{\alpha}}(t)=\prod_{i=1}^{m} l_{i}^{\alpha_{i}}(t) \quad \text { for all } t>0
$$

(and $l_{1}(t)=1+|\log t|, l_{i}(t)=l_{1}\left(l_{i-1}(t)\right)$ if $i>1$ ), then the Lorentz-Karamata space $L_{p, q ; b}(\Omega)$ is the generalized Lorentz-Zygmund space $L_{p, q, \boldsymbol{\alpha}}$ introduced in [9] and endowed with the (quasi-)norm $\|f\|_{p, q ; \boldsymbol{\alpha} ; \Omega}$, which in turn becomes the Lorentz-Zygmund space $L^{p, q}(\log L)^{\alpha_{1}}$ of Bennett and Rudnick [2] when $m=1$. If $\boldsymbol{\alpha}=(0, \ldots, 0)$, we obtain the Lorentz space $L_{p, q}(\Omega)$ endowed with the (quasi-)norm $\|\cdot\|_{p, q ; \Omega}$, which is just the Lebesgue space $L_{p}(\Omega)$ equipped with the (quasi-)norm $\|\cdot\|_{p ; \Omega}$ when $p=q$; if $p=q$ and $m=1$, we obtain the Zygmund space $L^{p}(\log L)^{\alpha_{1}}(\Omega)$ endowed with the (quasi-)norm $\|.\|_{p ; \alpha_{1} ; \Omega}$. 
The Bessel kernel $g_{\sigma}, \sigma>0$, is defined as that function on $\mathbf{R}^{n}$ whose Fourier transform is

$$
\widehat{g_{\sigma}}(\xi)=(2 \pi)^{-n / 2}\left(1+|\xi|^{2}\right)^{-\sigma / 2}, \quad \xi \in \mathbf{R}^{n},
$$

where the Fourier transform $\hat{f}$ of a function $f$ is given by

$$
\hat{f}(\xi)=(2 \pi)^{-n / 2} \int_{\mathbf{R}^{n}} e^{-i \xi \cdot x} f(x) d x .
$$

Let us summarize the basic properties of the Bessel kernel $g_{\sigma}$ :

$$
\begin{aligned}
& g_{\sigma} \text { is a positive, integrable function which is analytic except at the origin; } \\
& g_{\sigma}(x) \leq c_{1}|x|^{\sigma-n} e^{-c_{2}|x|} \text { for } 0<\sigma<n \text { and all } x \in \mathbf{R}^{n} \backslash\{0\} ; \\
& g_{\sigma}(x) \approx|x|^{\sigma-n} \text { as }|x| \rightarrow 0 \text { if } 0<\sigma<n ; \\
& \left|\frac{\partial}{\partial x_{j}} g_{\sigma}(x)\right| \leq c|x|^{\sigma-n-1} \text { for } 0<\sigma \leq n+1, j \in\{1, \ldots, n\} \text { and all } x \in \mathbf{R}^{n} \backslash\{0\} ; \\
& g_{\sigma}^{*}(t) \precsim t^{(\sigma-n) / n} e^{-c t^{1 / n}} \text { for } 0<\sigma<n \text { and all } t>0 .
\end{aligned}
$$

For the proof of (2.1)-(2.4) see [1], for (2.5) see [7].

Let $\sigma>0, p \in(1,+\infty), q \in[1,+\infty]$, and $b \in S V(0,+\infty)$. The Bessel-potential space $H^{\sigma} L_{p, q ; b}\left(\mathbf{R}^{n}\right)$ is defined to be

$$
\left\{u: u=g_{\sigma} * f, f \in L_{p, q ; b}\left(\mathbf{R}^{n}\right)\right\}
$$

and is equipped with the (quasi-)norm

$$
\|u\|_{\sigma ; p, q ; b}:=\|f\|_{p, q ; b} .
$$

For $\sigma=0$, we put

$$
g_{0} * f=f \quad \text { and } \quad H^{\sigma} L_{p, q ; b}\left(\mathbf{R}^{n}\right)=L_{p, q ; b}\left(\mathbf{R}^{n}\right) .
$$

When $m \in \mathbf{N}, \boldsymbol{\alpha}=\left(\alpha_{1}, \ldots, \alpha_{m}\right) \in \mathbf{R}^{m}$ and $b=\ell^{\boldsymbol{\alpha}}$, we obtain the logarithmic Bessel potential space $H^{\sigma} L_{p, q ;} \boldsymbol{\alpha}\left(\mathbf{R}^{n}\right)$, endowed with the (quasi-)norm $\|u\|_{\sigma ; p, q ; \boldsymbol{\alpha}}$ and considered in [9]. Note that if $\boldsymbol{\alpha}=(0, \ldots, 0)$, $H^{\sigma} L_{p, p ; \boldsymbol{\alpha}}\left(\mathbf{R}^{n}\right)$ is simply the (fractional) Sobolev space $H_{p}^{\sigma}\left(\mathbf{R}^{n}\right)$ of the order $\sigma$.

Let $\Omega$ be a domain in $\mathbf{R}^{n}$. The space of all scalar-valued (real or complex), bounded and continuous functions on $\Omega$ is denoted by $C_{B}(\Omega)$ and it is equipped with the $L_{\infty}(\Omega)$-norm.

Let $\mathcal{L}$ be the class of all continuous functions $\lambda:(0,1] \rightarrow(0,+\infty)$ which are increasing on some interval $(0, \delta)$, with $\delta=\delta_{\lambda} \in(0,1]$, and satisfy

$$
\lim _{t \rightarrow 0_{+}} \lambda(t)=0
$$

and

$$
\left\|\frac{t}{\lambda(t)}\right\|_{\infty ;(0, \delta)}<+\infty .
$$

Let $\lambda \in \mathcal{L}$ and let $\Omega$ be a domain in $\mathbf{R}^{n}$. The space $C^{0, \lambda(.)}(\bar{\Omega})$ consists of all those functions $f \in C_{B}(\Omega)$ for which the norm

$$
\left\|f\left|C^{0, \lambda(.)}(\bar{\Omega}) \|:=\sup _{x \in \Omega}\right| f(x) \mid+\sup _{\substack{x, y \in \Omega \\ 0<|x-y| \leq 1}} \frac{|f(x)-f(y)|}{\lambda(|x-y|)}\right.
$$

is finite. We refer to [19, Proposition 3.5] for an equivalent norm involving the modulus of smoothness.

If $\lambda(t)=t, t \in(0,1]$, and $\Omega=\mathbf{R}^{n}$, then $C^{0, \lambda(.)}(\bar{\Omega})$ coincides with the space $\operatorname{Lip}\left(\mathbf{R}^{n}\right)$ of the Lipschitz functions. Note also that if (2.8) does not hold, then $C^{0, \lambda(.)}(\bar{\Omega})$ consists only of constant functions on $\Omega$. 


\section{Statement of the results}

In this section we present the main results.

The next theorem concerns the superlimiting case and we assume that the order of smoothness is less than one.

Theorem 3.1 Let $0<\sigma<1, n / \sigma<p<+\infty, q \in(1,+\infty), b \in S V(0,+\infty)$, and let $\lambda \in \mathcal{L}$ be defined by

$$
\lambda(t)=t^{\sigma-n / p}\left[b\left(t^{n}\right)\right]^{-1} \text { for all } t>0 .
$$

Assume that $\Omega \subseteq \mathbf{R}^{n}$ is a nonempty domain.

(i) Then

$$
H^{\sigma} L_{p, q ; b}\left(\mathbf{R}^{n}\right) \hookrightarrow C^{0, \lambda(\cdot)}\left(\overline{\mathbf{R}^{n}}\right) .
$$

(ii) Let $n \geq 2$. If a function $\mu \in \mathcal{L}$ satisfies

$$
\liminf _{t \rightarrow 0_{+}} \frac{\mu(t)}{\lambda(t)}=0,
$$

then the embedding

$$
H^{\sigma} L_{p, q ; b}\left(\mathbf{R}^{n}\right) \hookrightarrow C^{0, \mu(\cdot)}(\bar{\Omega})
$$

does not hold.

(iii) Let $n \geq 2$. Then the embedding

$$
H^{\sigma} L_{p, q ; b}\left(\mathbf{R}^{n}\right) \hookrightarrow C^{0, \lambda(.)}(\bar{\Omega})
$$

is not compact.

The following theorem treats the limiting case and it is an analogue of Theorem 3.1 (i). However, the method used to prove that the embedding mentioned in Theorem 3.1 (i) is sharp and non-compact does not work in the limiting case. To prove that the limiting embedding from Theorem 3.2 below is sharp and non-compact, one needs a different approach. We return to this problem in another paper.

Theorem 3.2 Let $0<\sigma<n, p=n / \sigma, q \in(1,+\infty)$ and $b \in S V(0,+\infty)$ be such that

$$
\left\|t^{-1 / q^{\prime}}[b(t)]^{-1}\right\|_{q^{\prime} ;(0,1)}<+\infty .
$$

Let $\lambda \in \mathcal{L}$ be defined by

$$
\lambda(t)=\left(\int_{0}^{t^{n}}[b(\tau)]^{-q^{\prime}} \frac{d t}{t}\right)^{1 / q^{\prime}} \text { for all } t>0 .
$$

Then

$$
H^{\sigma} L_{p, q ; b}\left(\mathbf{R}^{n}\right) \hookrightarrow C^{0, \lambda(\cdot)}\left(\overline{\mathbf{R}^{n}}\right) .
$$

Remark 3.3 Note that there is no limiting embedding of form (3.4) in the classical situation when $b \equiv 1$ since then the assumption $\left\|t^{-1 / q^{\prime}}[b(t)]^{-1}\right\|_{q^{\prime} ;(0,1)}<+\infty$ is not satisfied. When $b \equiv 1$, then there are only the limiting embeddings (of the Trudinger or Brézis-Wainger type) into Lorentz-Zygmund spaces. Such embeddings are particular cases of $\left[15\right.$, Theorem 3.7 (i)], where it is assumed that $\left\|t^{-1 / q^{\prime}}[b(t)]^{-1}\right\|_{q^{\prime} ;(0,1)}=+\infty$.

Under the assumptions of Theorem 3.2, the functions from the space $H^{\sigma} L_{p, q ; b}\left(\mathbf{R}^{n}\right)$ are $\lambda(\cdot)$-Hölder continuous on $\mathbf{R}^{n}$. However, this Hölder continuity is a "weak one" since now the function $\lambda$ is a slowly varying function (which is a quite different situation from that of Theorem 3.1 (i)). For example, Theorem 3.2 implies that Sobolev-Orlicz space $W^{k} L^{n / k}(\log L)^{\alpha}\left(\mathbf{R}^{n}\right), k \in \mathbf{N}$ and $k<n$ (the Sobolev space modeled upon the Orlicz 
space $L^{n / k}(\log L)^{\alpha}\left(\mathbf{R}^{n}\right)=L_{\Phi}\left(\mathbf{R}^{n}\right)$, where the Young function satisfies $\left.\Phi(t)=\left[t(1+|\log t|)^{\alpha}\right]^{n / k}, t>0\right)$ is continuously embedded into the $\lambda(\cdot)$-Hölder class $C^{0, \lambda(\cdot)}\left(\overline{\mathbf{R}^{n}}\right)$ with

$$
\lambda(t)=(1+|\log t|)^{-\alpha+1-k / n} \text { for all } t>0,
$$

provided that $\alpha>1-k / n$ (the function $\lambda(t)$ tends to 0 as $t \rightarrow 0_{+}$more slowly than any function $t^{\varepsilon}$ with $\varepsilon>0)$. This illustrates the important role of the logarithmic term $(\log L)^{\alpha}$ involved in the Sobolev-Orlicz space $W^{k} L^{n / k}(\log L)^{\alpha}\left(\mathbf{R}^{n}\right)$. (By the classical results, the Sobolev space $W^{k, n / k}\left(\mathbf{R}^{n}\right)=W^{k} L_{n / k}\left(\mathbf{R}^{n}\right), k \in \mathbf{N}$ and $k<n$, is not even continuously embedded into the space $L_{\infty}(\Omega)$ for any domain $\Omega \subset \mathbf{R}^{n}$.) The embedding mentioned above (with $\lambda$ from (3.5)) should be also compared with the Brézis-Wainger type embedding $W^{k+1} L^{n / k}(\log L)^{\alpha}\left(\mathbf{R}^{n}\right) \hookrightarrow C^{0, \lambda(\cdot)}\left(\overline{\mathbf{R}^{n}}\right), k \in \mathbf{N}$ and $k<n$, where

$$
\lambda(t)=t(1+|\log t|)^{-\alpha+1-k / n} \text { for all } t>0,
$$

provided that $\alpha<1-k / n$ (which follows from [16, Theorem 3.2] or [9, Theorem 4.11]).

\section{Preliminaries for embeddings}

The next lemma generalizes [11, Lemma 2.5].

Lemma 4.1 Let $0<\sigma<n, n / \sigma<p<+\infty$ and $p<n /(\sigma-1)$ if $\sigma>1$. Let $q \in[1,+\infty]$ and $b \in S V(0,+\infty)$. Then, for all $h \in \mathbf{R}^{n}$ with $|h|>0$,

$$
\left\|\Delta_{h} g_{\sigma}\right\|_{p^{\prime}, q^{\prime} ; 1 / b} \precsim|h|^{\sigma-n / p}\left[b\left(|h|^{n}\right)\right]^{-1}{ }^{*}
$$

Proof. We use the ideas of [11, Lemma 2.5]. Put $B(r):=B_{n}(0, r)$ and $B^{C}(r):=\mathbf{R}^{n} \backslash B_{n}(0, r)$ for $r>0$. Let $h \in \mathbf{R}^{n}$ with $|h|>0$. Since

$$
\left\|\left(\Delta_{h} g_{\sigma}\right) \chi_{B(2|h|)}\right\|_{p^{\prime}, q^{\prime} ; 1 / b} \precsim 2\left\|g_{\sigma} \chi_{B(3|h|)}\right\|_{p^{\prime}, q^{\prime} ; 1 / b}
$$

using (2.5) and the fact that $\sigma / n-1 / p>0$, we obtain

$$
\begin{aligned}
\left\|\left(\Delta_{h} g_{\sigma}\right) \chi_{B(2|h|)}\right\|_{p^{\prime}, q^{\prime} ; 1 / b} & \precsim\left\|t^{1 / p^{\prime}-1 / q^{\prime}}[b(t)]^{-1} t^{\sigma / n-1}\right\|_{q^{\prime} ;\left(0, \omega_{n}(3|h|)^{n}\right)} \\
& =\left\|t^{\sigma / n-1 / p-1 / q^{\prime}}[b(t)]^{-1}\right\|_{q^{\prime} ;\left(0, \omega_{n}(3|h|)^{n}\right)} \\
& \approx|h|^{\sigma-n / p}\left[b\left(|h|^{n}\right)\right]^{-1} .
\end{aligned}
$$

Now, we derive an estimate in the exterior of the ball $B(2|h|)$. The inequality

$$
\left|\Delta_{h} g_{\sigma}(x)\right| \leq|h| \sum_{j=1}^{n} \int_{0}^{1}\left|\frac{\partial}{\partial x_{j}} g_{\sigma}(x+\tau h)\right| d \tau \quad \text { for all } \quad x \in \mathbf{R}^{n} \backslash\{0\},
$$

together with the obvious estimate

$$
\frac{1}{2}|x| \leq|x+\tau h| \leq \frac{3}{2}|x| \quad \text { if } \quad \tau \in[0,1] \quad \text { and } \quad x \in B^{C}(2|h|)
$$

and (2.4), yields

$$
\left|\Delta_{h} g_{\sigma}(x) \chi_{B^{C}(2|h|)}(x)\right| \precsim|h||x|^{\sigma-n-1} \chi_{B^{C}(|h|)}(x) \quad \text { for all } \quad x \in \mathbf{R}^{n} \backslash\{0\} .
$$

Putting

$$
F(x)=|x|^{\sigma-n-1} \chi_{B^{C}(|h|)}(x), \quad x \in \mathbf{R}^{n} \backslash\{0\},
$$

\footnotetext{
${ }^{*}$ For each $h \in \mathbf{R}^{n}$, the first difference operator $\Delta_{h}$ is defined on functions on $\mathbf{R}^{n}$ by $\Delta_{h} f(x):=f(x+h)-f(x), x \in \mathbf{R}^{n}$.
} 
and taking into account that $\sigma-n-1<0$, we can easily see that

$$
F^{*}(t)=\left(|h|^{n}+\frac{t}{\omega_{n}}\right)^{(\sigma-n-1) / n} \text { for all } t>0 .
$$

This and (4.4) imply that

$$
\left\|\left(\Delta_{h} g_{\sigma}\right) \chi_{B^{C}(2|h|)}\right\|_{p^{\prime}, q^{\prime} ; 1 / b} \precsim|h|\left\|t^{1 / p^{\prime}-1 / q^{\prime}}[b(t)]^{-1} F^{*}(t)\right\|_{q^{\prime} ;(0,+\infty)}=|h|\left(N_{1}+N_{2}\right),
$$

where $N_{1}=\left\|t^{1 / p^{\prime}-1 / q^{\prime}}[b(t)]^{-1} F^{*}(t)\right\|_{q^{\prime} ;\left(0,|h|^{n}\right)}$ and $N_{2}=\left\|t^{1 / p^{\prime}-1 / q^{\prime}}[b(t)]^{-1} F^{*}(t)\right\|_{\left.q^{\prime} ;|h|^{n},+\infty\right)}$. Since $\sigma-n-1<0,(4.5)$ shows that $F^{*}(t) \leq|h|^{\sigma-n-1}$ for all $t>0$. This and the fact that $p>1$ yield

$$
N_{1} \leq|h|^{\sigma-n-1}\left\|t^{1 / p^{\prime}-1 / q^{\prime}}[b(t)]^{-1}\right\|_{q^{\prime} ;\left(0,|h|^{n}\right)} \approx|h|^{\sigma-1-n / p}\left[b\left(|h|^{n}\right)\right]^{-1} .
$$

Again, since $\sigma-n-1<0$, (4.5) shows that $F^{*}(t) \precsim t^{(\sigma-n-1) / n}$ for all $t>0$. Hence, because $\sigma-1-n / p<0$,

$$
\begin{aligned}
N_{2} & \precsim\left\|t^{1 / p^{\prime}-1 / q^{\prime}}[b(t)]^{-1} t^{(\sigma-n-1) / n}\right\|_{q^{\prime} ;\left(|h|^{n},+\infty\right)} \\
& =\left\|t^{\left(\sigma / n-1 / n-1 / p-1 / q^{\prime}\right.}[b(t)]^{-1}\right\|_{q^{\prime} ;\left(|h|^{n},+\infty\right)} \\
& \approx|h|^{\sigma-1-n / p}\left[b\left(|h|^{n}\right)\right]^{-1} .
\end{aligned}
$$

Estimates (4.6)-(4.8) imply that

$$
\left\|\left(\Delta_{h} g_{\sigma}\right) \chi_{B^{C}(2|h|)}\right\|_{p^{\prime}, q^{\prime} ; 1 / b} \precsim|h|^{\sigma-n / p}\left[b\left(|h|^{n}\right)\right]^{-1} .
$$

The result is a consequence of (4.2) and (4.9).

The next lemma generalizes [11, Lemma 2.6].

Lemma 4.2 Let $0<\sigma<n$ and $p=n / \sigma$. Let $q \in[1,+\infty]$ and $b \in S V(0,+\infty)$ be such that

$$
\left\|t^{-1 / q^{\prime}}[b(t)]^{-1}\right\|_{q^{\prime} ;(0,1)}<+\infty .
$$

Then, for all $h \in \mathbf{R}^{n}$ with $|h|>0$,

$$
\left\|\Delta_{h} g_{\sigma}\right\|_{p^{\prime}, q^{\prime} ; 1 / b} \precsim\left\|t^{-1 / q^{\prime}}[b(t)]^{-1}\right\|_{q^{\prime} ;\left(0,|h|^{n}\right)^{n}} .
$$

Pro of. We proceed as in the proof of Lemma 4.1. First, note that assumption (4.10) implies that the function $\tau \mapsto\left\|t^{-1 / q^{\prime}}[b(t)]^{-1}\right\|_{q^{\prime} ;(0, \tau)}$ belongs to $S V(0,+\infty)$. Using this fact, (2.5) and the identity $\sigma / n-1 / p=0$, we obtain instead of (4.2) that

$$
\left\|\Delta_{h} g_{\sigma} \chi_{B(2|h|)}\right\|_{p^{\prime}, q^{\prime} ; 1 / b} \precsim\left\|t^{-1 / q^{\prime}}[b(t)]^{-1}\right\|_{q^{\prime} ;\left(0, \omega_{n}(3|h|)^{n}\right)} \approx\left\|t^{-1 / q^{\prime}}[b(t)]^{-1}\right\|_{q^{\prime} ;\left(0,|h|^{n}\right)^{n}} .
$$

Since the estimates of the quantities $N_{1}$ and $N_{2}$ remain true, we again have (4.9), that is,

$$
\left\|\left(\Delta_{h} g_{\sigma}\right) \chi_{B^{C}(2|h|)}\right\|_{p^{\prime}, q^{\prime} ; 1 / b} \precsim\left[b\left(|h|^{n}\right)\right]^{-1} .
$$

Moreover, since $b \in S V(0,+\infty)$,

$$
\left[b\left(|h|^{n}\right)\right]^{-1} \precsim\left\|t^{-1 / q^{\prime}}[b(t)]^{-1}\right\|_{q^{\prime} ;\left(0,|h|^{n}\right)} .
$$

Consequently, the result follows from (4.12) and (4.13). 


\section{Preliminaries for sharpness and non-compactness of the embeddings}

To prove sharpness and non-compactness of the embeddings, we need to construct suitable test functions. We use the ideas of [10] and [12]. Throughout this section we shall assume that $\mathcal{G}$ is a function on $(0,1]$ with the following properties:

$$
\begin{aligned}
& \mathcal{G} \text { is positive and continuous on }(0,1] \text {; } \\
& t \mathcal{G}(t) \text { is non-increasing on }\left(0, s_{0}\right] \text {, where } s_{0} \in(0,1] \text { is a fixed number; } \\
& \mathcal{G}(t / 2) \precsim \mathcal{G}(t), \quad t \in(0,1]
\end{aligned}
$$

(note that the assumption (5.2) is stronger than (4.2) of [10]). Let $\varphi \in C_{0}^{\infty}(\mathbf{R})$ be a nonnegative function such that $\int_{\mathbf{R}} \varphi(t) d t=1$ and $\operatorname{supp} \varphi=[-1,1]$, and define the function $\varphi_{\varepsilon}, \varepsilon>0$, by $\varphi_{\varepsilon}(t):=\frac{1}{\varepsilon} \varphi\left(\frac{t}{\varepsilon}\right)$ for all $t \in \mathbf{R}$. We now use $\varphi$ to assign to the function $\mathcal{G}$ a family of functions $\left\{\mathcal{G}_{s}\right\}$ as in [10]. We extend $\mathcal{G}$ by zero outside the interval $(0,1]$ and, for each $s \in(0,1)$, define the function $\mathcal{G}_{s}$ by

$$
\mathcal{G}_{s}(t):=\left(\chi_{[s,+\infty)} \psi \mathcal{G}\right) * \varphi_{\frac{s}{4}}(t), \quad t \in \mathbf{R},
$$

where $\psi \in C_{0}^{\infty}(\mathbf{R})$ is given by $\psi=\chi_{\left[-2+\frac{1}{16}, \frac{3}{4}-\frac{1}{16}\right]} * \varphi_{\frac{1}{16}}$.

Some properties of $\mathcal{G}_{s}, s \in(0,1 / 4)$, are summarized in the next lemma due to Edmunds, Gurka and Opic [10, Lemma 4.1].

Lemma 5.1 If $s \in\left(0, \frac{1}{4}\right)$ and the functions $\mathcal{G}_{s}$ are defined by (5.4) (with $\mathcal{G}$ satisfying (5.1)-(5.3)), then

$$
\mathcal{G}_{s} \in C_{0}^{\infty}(\mathbf{R}), \quad \operatorname{supp} \mathcal{G}_{s} \subset\left[\frac{s}{2}, 1\right] \quad \text { and } \quad \mathcal{G}_{s} \geq 0 .
$$

Moreover, there are positive constants $C_{1}$ and $C_{2}$ (independent of $s$ and $t$ ) such that

$$
\begin{array}{ll}
\mathcal{G}_{s}(t) \leq C_{1} \mathcal{G}(t) \chi_{\left[\frac{s}{2}, 1\right]}(t), & t \in(0,1], \\
\mathcal{G}_{s}(t) \geq C_{2} \mathcal{G}(t), & t \in\left[2 s, \frac{1}{2}\right] .
\end{array}
$$

Now, as in [12], the family $\left\{\mathcal{G}_{s}\right\}$ is used to define test functions $u_{s}$. For any $s \in(0,1 / 4)$, let $a_{s}$ be a positive number and let $\mathcal{G}_{s}$ be the function given by (5.4). We put

$$
u_{s}(x):=x_{1}\left(g_{\sigma} * h_{s}\right)(x), \quad x=\left(x_{1}, \ldots, x_{n}\right) \in \mathbf{R}^{n},
$$

where

$$
h_{s}(x):=a_{s} \mathcal{G}_{s}(|x|) \text { for all } x \in \mathbf{R}^{n} .
$$

In order to prove that the functions $u_{s}$ belong to the source space of our embeddings, we need the following preliminary results.

Lemma 5.2 ([12, Lemma 2]) Let h belong to the Schwartz space $\mathcal{S}, \sigma \geq 0, j \in\{1, \ldots, n\}$ and let $\mathcal{R}_{j}$ be the Riesz transform. Then there exists a finite measure $\nu$ on $\mathbf{R}^{n}$ such that, for any $x=\left(x_{1}, \ldots, x_{n}\right) \in \mathbf{R}^{n}$,

$$
x_{j}\left(g_{\sigma} * h\right)(x)=-\sigma(2 \pi)^{-n / 2}\left[g_{\sigma} *\left(\mathcal{R}_{j}\left(\nu * g_{1} * h\right)\right)\right](x)+\left[g_{\sigma} *\left(y_{j} h(y)\right)\right](x) .
$$

The next lemma extends [10, Corollary 4.12].

Lemma 5.3 Let $1<p<+\infty, 1 \leq q \leq+\infty, b \in S V(0,+\infty)$, and let $\nu$ be the finite measure from Lemma 5.2. Then, for all $f \in L_{p, q ; b}\left(\mathbf{R}^{n}\right)$,

$$
\begin{aligned}
\left\|g_{\sigma} * f\right\|_{p, q ; b} & \precsim\|f\|_{p, q ; b}, \quad \sigma \geq 0, \\
\left\|\mathcal{R}_{j} f\right\|_{p, q ; b} & \precsim\|f\|_{p, q ; b}, \quad j=1, \ldots, n, \\
\|\nu * f\|_{p, q ; b} & \precsim\|f\|_{p, q ; b} .
\end{aligned}
$$

Proof. We use the boundedness of the operators $g_{\sigma} * f, \mathcal{R}_{j} f$ and $\nu * f$ in $L_{r}\left(\mathbf{R}^{n}\right), r \in(1,+\infty)$, and apply [16, Lemma 4.4]. 
We shall need as well the next result.

Lemma 5.4 Let $n \geq 2, n /(n-1)<p<\infty, q \in[1,+\infty], 1 / p=1 / \tilde{p}-1 / n$, and let $b \in S V(0,+\infty)$. Then, for all $f \in L_{\tilde{p}, q ; b}\left(\mathbf{R}^{n}\right)$,

$$
\left\|g_{1} * f\right\|_{p, q ; b} \precsim\|f\|_{\tilde{p}, q ; b} .
$$

Proof. The assumption $n /(n-1)<p<+\infty$ implies that $\tilde{p} \in(1, n)$. The result is now a consequence of [15, Theorem 3.1 (i)], with $\sigma=1$ and $r=q$.

Lemma 5.5 Let $p \in(1,+\infty), q \in[1,+\infty]$ and $b \in S V(0,+\infty)$. Let $g$ be a positive function which is continuous in $(0,1]$, non-increasing in some interval $\left(0, r_{0}\right) \subset(0,1]$ and such that $g(t / 2) \precsim g(t), t \in(0,1)$. Then, for all $s \in\left(0, \frac{1}{4}\right)$,

$$
\left\|g(|y|) \chi_{\left(\frac{s}{2}, 1\right)}(|y|)\right\|_{p, \infty ; b} \leq \sup _{t \in[s, 1)} t^{n / p} g(t) b\left(t^{n}\right),
$$

and, if $q \in[1,+\infty)$,

where

$$
\left\|g(|y|) \chi_{\left(\frac{s}{2}, 1\right)}(|y|)\right\|_{p, q ; b} \leq\left(V_{1}(s)+V_{2}(s)\right),
$$

$$
V_{1}(s)=\left(\int_{s}^{1}\left[t^{n / p} g(t) b\left(t^{n}\right)\right]^{q} \frac{d t}{t}\right)^{1 / q} \quad \text { and } \quad V_{2}(s)=s^{n / p} g(s) b\left(s^{n}\right) .
$$

Proof. See the proof of [15, Lemma 4.3]; see as well the proof of [8, Lemma 4.1].

We shall make use of the next lemma which generalizes [12, Lemma 6].

Lemma 5.6 Let $\sigma \geq 0, n \geq 2, n /(n-1)<p<+\infty, q \in[1,+\infty)$ and $b \in S V(0,+\infty)$. Then the functions $u_{s}, s \in\left(0, \frac{1}{4}\right)$, defined by (5.8) (with $\mathcal{G}$ from (5.1)-(5.3)), satisfy

where

$$
\left\|u_{s}\right\|_{\sigma ; p, q ; b} \leq a_{s}\left(W_{1}(s)+W_{2}(s)\right),
$$

$$
W_{1}(s)=\left(\int_{s}^{1}\left[t^{n / p+1} \mathcal{G}(t) b\left(t^{n}\right)\right]^{q} \frac{d t}{t}\right)^{1 / q} \quad \text { and } \quad W_{2}(s)=s^{n / p+1} \mathcal{G}(s) b\left(s^{n}\right)
$$

Pr o of. We follow the proof of [12, Lemma 6]. It follows from (5.5) that $u_{s} \in S\left(\mathbf{R}^{n}\right)$. Thus, by Lemma 5.2, (2.6) and (5.8) we obtain the estimate

$$
\begin{aligned}
\left\|u_{s}\right\|_{\sigma ; p, q ; b} \precsim \sigma(2 \pi)^{-n / 2}\left\|g_{\sigma} *\left(\mathcal{R}_{1}\left(\nu * g_{1} * h_{s}\right)\right)\right\|_{\sigma ; p, q ; b}+\left\|g_{\sigma} *\left(y_{j} h_{s}(y)\right)\right\|_{\sigma ; p, q ; b} \\
=\sigma(2 \pi)^{-n / 2}\left\|\mathcal{R}_{1}\left(\nu * g_{1} * h_{s}\right)\right\|_{p, q ; b}+\left\|y_{j} h_{s}(y)\right\|_{p, q ; b} .
\end{aligned}
$$

Applying Lemma 5.3, Lemma 5.4, (5.9) and (5.6) to the first term, we obtain

$$
\left\|\mathcal{R}_{1}\left(\nu * g_{1} * h_{s}\right)\right\|_{p, q ; b} \precsim\left\|g_{1} * h_{s}\right\|_{p, q ; b} \precsim\left\|h_{s}\right\|_{\tilde{p}, q ; b} \precsim a_{s}\left\|\mathcal{G}(|y|) \chi_{\left[\frac{s}{2}, 1\right]}(|y|)\right\|_{\tilde{p}, q ; b} .
$$

Moreover, Lemma 5.5, with $g=\mathcal{G}$ (which satisfies the assumptions of Lemma 5.5), and the identity $n / \tilde{p}=$ $n / p+1$ yield

$$
\left\|\mathcal{G}(|y|) \chi_{\left[\frac{s}{2}, 1\right]}(|y|)\right\|_{\tilde{p}, q ; b} \precsim\left(W_{1}(s)+W_{2}(s)\right) .
$$

Hence,

$$
\sigma(2 \pi)^{-n / 2}\left\|\mathcal{R}_{1}\left(\nu * g_{1} * h_{s}\right)\right\|_{p, q ; b} \precsim a_{s}\left(W_{1}(s)+W_{2}(s)\right) .
$$


Again, by (5.6), (5.9) and Lemma 5.5, with $g(t)=t \mathcal{G}(t)$ (which also satisfies the assumptions of Lemma 5.5), we have

$$
\begin{aligned}
\left\|y_{1} h_{s}(y)\right\|_{p, q ; b} & \leq\left\||y| h_{s}(y)\right\|_{p, q ; b} \\
& \precsim a_{s}\left\||y| \mathcal{G}(|y|) \chi_{\left[\frac{s}{2}, 1\right]}(|y|)\right\|_{\tilde{p}, q ; b} \\
& \precsim a_{s}\left(W_{1}(s)+W_{2}(s)\right) .
\end{aligned}
$$

The result now follows from (5.13), (5.14) and (5.15).

To prove sharpness and non-compactness, we need as well the next assertion.

Lemma 5.7 (i) If $\sigma \in(0, n)$, then there exists a positive constant $c$ such that for every $s \in\left(0, \frac{1}{4}\right)$ and $x=$ $(t, 0, \ldots, 0) \in \mathbf{R}^{n}, t \in\left[2 s, \frac{1}{2}\right]$,

$$
\left|u_{s}(x)-u_{s}(0)\right| \geq \operatorname{cta} \int_{t}^{1 / 2} \tau^{\sigma-1} \mathcal{G}(\tau) d \tau
$$

(ii) Let $\sigma \in(0, n), S \in\left(0, \frac{1}{4}\right)$. Suppose that the numbers $a_{s}$ from (5.9) are bounded, i.e.,

$$
a_{s} \leq c \text { for all } s \in\left(0, \frac{1}{4}\right) \text { with some } c \in(0,+\infty) .
$$

Moreover, assume (in addition to (5.1)-(5.3)) that the function $\mathcal{G}$ and the numbers $a_{s}$ satisfy

$$
a_{s} \int_{2 s}^{S / 2} t^{\sigma-1} \mathcal{G}(t) d t \longrightarrow+\infty \quad \text { as } \quad s \longrightarrow 0_{+} .
$$

Then there exist $\varepsilon=\varepsilon(\sigma) \in\left(0, \frac{1}{2}\right), s_{1}=s_{1}(S) \in\left(0, \frac{S}{4}\right)$ and a positive constant $c$ (independent of $S$ and $s_{1}$ ) such that

$$
\left|\left[u_{s}(x)-u_{S}(x)\right]-\left[u_{s}(0)-u_{S}(0)\right]\right| \geq \operatorname{csa} a_{s} \int_{2 s}^{S / 2} t^{\sigma-1} \mathcal{G}(t) d t
$$

for every $s \in\left(0, s_{1}\right)$ and $x=(\varepsilon s, 0, \ldots, 0) \in \mathbf{R}^{n}$.

Pro of. The result of (i) is an adaptation of [16, Lemma 4.3 (ii)]. The assertion in (ii) immediately follows from [10, Lemma 4.5] because $u_{s}(0)=u_{S}(0)=0$.

\section{Proofs of the main results}

Proof of Theorem 3.1 .

Step 1. Put $X=L_{p, q ; b}\left(\mathbf{R}^{n}\right)$. Then its associate space $X^{\prime}$ is given by $X^{\prime}=L_{p^{\prime}, q^{\prime} ; 1 / b}\left(\mathbf{R}^{n}\right)$ (cf. [20, Theorem 3.1] with $\gamma_{b}$ replaced by $b$ and $\gamma_{1 / b}$ by $1 / b$, respectively). Let $u \in H^{\sigma} X$ and $\|u\|_{H^{\sigma} X} \leq 1$. Then there is a function $f \in X$ such that $u=g_{\sigma} * f$ and $\|f\|_{X}=\|u\|_{H^{\sigma} X} \leq 1$. Therefore, by using the Hölder inequality (cf. [3, Corollary II.4.5]),

$$
|u(x+h)-u(x)|=\left|\int_{\mathbf{R}^{n}} f(y) \Delta_{h} g_{\sigma}(x-y) d y\right| \precsim\|f\|_{X}\left\|\Delta_{h} g_{\sigma}\right\|_{p^{\prime}, q^{\prime} ; 1 / b} \leq\left\|\Delta_{h} g_{\sigma}\right\|_{p^{\prime}, q^{\prime} ; 1 / b} .
$$

This, together with Lemma 4.1, yields

$$
|u(x+h)-u(x)| \precsim \lambda(|h|) \text { for all } x \in \mathbf{R}^{n} \text { and } h \in \mathbf{R}^{n} \text { with }|h|>0 .
$$

Since also $H^{\sigma} X \hookrightarrow C_{B}\left(\mathbf{R}^{n}\right)$ by [21, Proposition 5.6], the proof of part (i) now follows.

Step 2. We shall assume without loss of generality that $B_{n}(0,1) \subset \Omega$. Let $s \in\left(0, \frac{1}{4}\right)$ and $\gamma<0$. Define the function $\mathcal{G}$ by

$$
\mathcal{G}(t)=t^{\gamma-1-n / p}\left[b\left(t^{n}\right)\right]^{-1}, \quad t \in(0,1]
$$


and put

$$
a_{s}=s^{-\gamma}
$$

The function $\mathcal{G}$ satisfies (5.1)-(5.3). Let us consider the functions $u_{s}, s \in\left(0, \frac{1}{4}\right)$, defined by (5.8). By Lemma 5.6, (6.2) and (6.3), for all $s \in\left(0, \frac{1}{4}\right)$,

$$
\left\|u_{s}\right\|_{\sigma ; p, q ; b} \precsim a_{s}\left(W_{1}(s)+W_{2}(s)\right) \approx s^{-\gamma}\left(\left(\int_{s}^{1} t^{\gamma q-1} d t\right)^{1 / q}+s^{\gamma}\right) \approx s^{-\gamma} s^{\gamma}=1 .
$$

By Lemma 5.7 (i), (6.2) and (6.3), there exists a positive constant $c$ such that

$$
\left|u_{s}(x)-u_{s}(0)\right| \geq 2 c s^{1-\gamma} \int_{2 s}^{1 / 2} t^{\sigma-1+\gamma-1-n / p}\left[b\left(t^{n}\right)\right]^{-1} d t \succsim s^{\sigma-n / p}\left[b\left(s^{n}\right)\right]^{-1}
$$

for every $s \in\left(0, \frac{1}{4}\right)$ and $x=(2 s, 0, \ldots, 0)$.

Furthermore, if we take $S \in\left(0, \frac{1}{4}\right)$, we can see that the conditions (5.16) and (5.17) also hold. Indeed, $a_{s}=s^{-\gamma} \precsim 1$ for all $s \in\left(0, \frac{1}{4}\right)$ because $\gamma<0$. Moreover, since $\sigma-n / p-1<0$ and $\gamma<0$, we have, for all sufficiently small $s$,

$$
\begin{aligned}
a_{s} \int_{2 s}^{S / 2} t^{\sigma-1} \mathcal{G}(t) d t & \approx a_{s} \int_{2 s}^{S / 2} t^{\sigma-1+\gamma-1-n / p}\left[b\left(t^{n}\right)\right]^{-1} d t \\
& \approx s^{-\gamma+\sigma-1+\gamma-n / p}\left[b\left(s^{n}\right)\right]^{-1} \\
& \approx s^{\sigma-1-n / p}\left[b\left(s^{n}\right)\right]^{-1},
\end{aligned}
$$

which tends to $+\infty$ as $s \rightarrow 0_{+}$. Hence, by Lemma 5.7 (ii), (6.2) and (6.3), there exist $\varepsilon=\varepsilon(\sigma) \in\left(0, \frac{1}{2}\right)$, $s_{1}=s_{1}(S) \in\left(0, \frac{S}{4}\right)$ and a positive constant $c$ (independent of $S$ and $s_{1}$ ) such that, for every $s \in\left(0, s_{1}\right)$ and $x=(\varepsilon s, 0, \ldots, 0)$,

$$
\left|\left[u_{s}(x)-u_{S}(x)\right]-\left[u_{s}(0)-u_{S}(0)\right]\right| \geq c s^{1-\gamma} \int_{2 s}^{S / 2} t^{\sigma-1} \mathcal{G}(t) d t \geq c_{1} s^{\sigma-n / p}\left[b\left(s^{n}\right)\right]^{-1},
$$

with a positive constant $c_{1}$ independent of $S$ and $s_{1}$.

Step 3. Let $\lambda$ be the function defined by (3.1). Since $b \in S V(0,+\infty)$, we have, for any fixed $k \in(0,+\infty)$,

$$
\lambda(k t) \approx \lambda(t), \quad t \in(0,1]
$$

Let us assume that (3.2) and (3.3) hold. Then, by (6.4), (6.5) (with $x=(2 s, 0, \ldots, 0))$ and (6.7), we obtain

$$
1 \succsim\left\|u_{s}\right\|_{\sigma ; p, q ; b} \succsim\left\|u_{s} \mid C^{0, \mu(.)}(\bar{\Omega})\right\| \geq \frac{\left|u_{s}(x)-u_{s}(0)\right|}{\mu(2 s)} \succsim \frac{s^{\sigma-n / p}\left[b\left(s^{n}\right)\right]^{-1}}{\mu(2 s)} \approx \frac{\lambda(2 s)}{\mu(2 s)}
$$

for all $s \in\left(0, \frac{1}{4}\right)$, which contradicts assumption (3.2). The proof of part (ii) is complete.

Step 4. Take $S \in\left(0, \frac{1}{4}\right)$ fixed. Let $\lambda$ be the function defined by (3.1). Then, (6.6) (with $\left.x=(\varepsilon s, 0, \ldots, 0)\right)$ and (3.1) yield, for every sufficiently small positive $s$,

$$
\left\|\left(u_{s}-u_{S}\right) \mid C^{0, \lambda(.)}(\bar{\Omega})\right\| \geq \frac{\left|\left[u_{s}(x)-u_{S}(x)\right]-\left[u_{s}(0)-u_{S}(0)\right]\right|}{\lambda(\varepsilon s)} \geq c_{1} \frac{\lambda(s)}{\lambda(\varepsilon s)} \geq c_{2},
$$

with $c_{2}$ a positive constant independent of $s$ and $S$. Therefore, if we consider the sequence $\left\{u_{1 / k}\right\}_{k=k_{0}}^{+\infty}$, with $k_{0}$ sufficiently large, then, by (6.4), this sequence is bounded in $H^{\sigma} L_{p, q ; b}\left(\mathbf{R}^{n}\right)$. However, by (6.8), it has no Cauchy subsequence in $C^{0, \lambda(.)}(\bar{\Omega})$. The proof of part (iii) is complete.

Proof of The ore m 3.2. The proof is similar to that of Theorem 3.1 (i). One applies Lemma 4.2 instead of Lemma 4.1. 
Acknowledgements This work was supported by Grant No. 201/05/2033 of the Grant Agency of the Czech Republic, by the Institutional Research plan No. AV0Z10190503 of the Academy of Sciences of the Czech Republic and by Centre of Mathematics of the University of Coimbra.

\section{References}

[1] N. Aronszajn, F. Mulla, and P. Szeptycki, On spaces of potentials connected with $L^{p}$ classes, Part I, Ann. Inst. Fourier (Grenoble) 13, No. 2, 211-306 (1963).

[2] C. Bennett and K. Rudnick, On Lorentz-Zygmund spaces, Dissertationes Math. (Rozprawy Mat.) 175, 1-72 (1980).

[3] C. Bennett and R. Sharpley, Interpolation of Operators, Pure and Applied Mathematics Vol. 129 (Academic Press, New York, 1988).

[4] N. H. Bingham, C. M. Goldie, and J. L. Teugels, Regular Variation (Cambridge University Press, Cambridge, 1987).

[5] H. Brézis and S. Wainger, A note on limiting cases of Sobolev embeddings, Comm. Partial Differential Equations 5, $773-789$ (1980).

[6] A. P. Calderón, Lebesgue spaces of differentiable functions and distributions, in: Partial Differential Equations, Proceedings of Symposia in Pure Mathetics Vol. 4 (Amer. Math. Soc., Providence, RI, 1961), pp. 33-49.

[7] D. E. Edmunds, P. Gurka, and B. Opic, Double exponential integrability, Bessel potentials and embedding theorems, Studia Math. 115, 151-181 (1995).

[8] D. E. Edmunds, P. Gurka, and B. Opic, Sharpness of embeddings in logarithmic Bessel potential spaces, Proc. Roy. Soc. Edinburgh Sect. A 126, 995-1009 (1996).

[9] D. E. Edmunds, P. Gurka, and B. Opic, On embeddings of logarithmic Bessel potential spaces, J. Funct. Anal. 146, No. 1, 116-150 (1997).

[10] D. E. Edmunds, P. Gurka, and B. Opic, Optimality of embeddings of logarithmic Bessel potential spaces, Q. J. Math. 51, No. 2, 185-209 (2000).

[11] D. E. Edmunds, P. Gurka, and B. Opic, Compact and continuous embeddings of logarithmic Bessel potential spaces, Studia Math. 168, No. 3, 229-250 (2005).

[12] D. E. Edmunds, P. Gurka, and B. Opic, Non-compact and sharp embeddings of logarithmic Bessel potential spaces into Hölder-type spaces, Z. Anal. Anwend. 25, 73-80 (2006).

[13] D. E. Edmunds, R. Kerman, and L. Pick, Optimal Sobolev embeddings involving rearrangement-invariant quasinorms, J. Funct. Anal. 170, 307-355 (2000).

[14] W. D. Evans and B. Opic, Real interpolation with logarithmic functors and reiteration, Canad. J. Math. 52, 920-960 (2000).

[15] A. Gogatishvili, J. S. Neves, and B. Opic, Optimality of embeddings of Bessel-potential-type spaces into LorentzKaramata spaces, Proc. Roy. Soc. Edinburgh Sect. A 134, 1127-1147 (2004).

[16] A. Gogatishvili, J. S. Neves, and B. Opic, Optimality of embeddings of Bessel-potential-type spaces into generalized Hölder spaces, Publ. Math. 49, 297-327 (2005).

[17] A. Gogatishvili, B. Opic, and W. Trebels, Limiting reiteration for real interpolation with slowly varying functions, Math. Nachr. 278, 86-107 (2005).

[18] V. Marić, Regular variation and differential equations, Lecture Notes in Mathematics Vol. 1726 (Springer-Verlag, Berlin, 2000).

[19] J. S. Neves, Extrapolation results on general Besov-Hölder-Lipschitz spaces, Math. Nachr. 230, 117-141 (2001).

[20] J. S. Neves, Lorentz-Karamata spaces, Bessel and Riesz potentials and embeddings, Dissertationes Math. (Rozprawy Mat.) 405, 1-46 (2002).

[21] J. S. Neves, Spaces of Bessel-potential type and embeddings: the super-limiting case, Math. Nachr. 265, 68-86 (2004).

[22] A. Zygmund, Trigonometric Series, Vol. I (Cambridge University Press, Cambridge, 1957). 